\title{
Hybrid of GPON and XGPON for Splitting Ratio of 1:64
}

\author{
Nana Rachmana Syambas and Rahadian Farizi \\ Telecommunication Research Group, School of Electrical and Informatics Engineering, ITB \\ Jl. Ganesha No.10 Bandung 40132 Indonesia \\ nana@stei.itb.ac.id
}

\begin{abstract}
In the last mile of telecommunication network technology, optical transmission network has been deployed using the Fiber-to-the Home (FTTH) technology to provide broadband user demands. Evolution of the FTTH network change very rapidly, causing an implementation of existing and new technology should be in coexistence or called in hybrid mode. Optical distribution networks consist of more than $60 \%$ of the total investments in deploying Passive Optical Networks (PON). So, it is essential for the evolution of PON to cooperation with the existing networks. This paper discusses compatible hybrid application of the Gigabit Passive Optical Network (GPON) and the 10-Gigabit-capable Passive Optical Network (XGPON) for splitting ratio of 1:64. The performance of the hybrid network system is analyzed using The Opti-system software tool. The transmitter, the receiver and the transmission channel are set in compliance to the ITU-T standards series G.984. A laser component with NRZ modulation scheme and the transmitter power is adjusted to maximum power. The fiber optic cable uses the Single Mode Fiber (SMF) and the bit rate used in the GPON is 2.5 Gbps downstream and 1.25 Gbps upstream, while on the XGPON the bit rate are $10 \mathrm{Gbps}$ downstream and $2.5 \mathrm{Gbps}$ upstream. The result of experiment by simulation shows that the performance of hybrid GPON and XGPON technology for 1:64 splitting ratio is suitable to be implemented in FTTH to provide broadband services. It means that the migration from GPON to XGPON can be done smoothly via hybrid mode to keep safe existing investment of Telco operators.
\end{abstract}

Keywords: Fiber-to-the-Home, GPON, XGPON, Hybrid.

\section{Introduction}

Fiber-to-the-Home (FTTH) has been widely used as a fixed broadband access technology, with the optical fiber as the media to the network from home environment in urban areas. In the last decade FTTH continues to grow significantly. Users are estimated to have reached more than 150 millions in the world mostly reside in Asia-Pacific region, North America and Europe. Recently in Indonesia, government has a program to replace copper cables with optical cables to every household mainly in big city and expected in the next future completion. The penetration of copper and fiber optic channels at home is still lower than $15 \%$. That means there are more than $85 \%$ of homes that are not covered by the copper cable or optical fiber. Therefore, FTTH will play an important role in connecting the house with ICT applications which will accelerate the growth of the national economy. The agenda of the digital economy is a priority for Indonesia and will be the backbone of the country's economic growth. However, broadband users in Indonesia reached less than 4.3 million and a new fixed broadband penetration is less than $7 \%$ in the whole country. So, there is a very big gap in the market to be filled.

FTTH networks are a Passive Optical Network (PON). It uses point-to-multipoint fiber configuration to the end users with optical splitters to split a single optical fiber to serve multiple end-points. FTTH is intensively deployed to make great progress in recent years in Indonesia, so certainly the needs of broadband services will increase in the next future. The broadband services and co-operation with existing technologies should be done by Telcos to guide implementation of new PON technologies. Telcos are trying to increase revenue by

Received: November $23^{\text {rd }}, 2016$. Accepted: March $23^{\text {rd }}, 2017$

DOI: 10.15676/ijeei.2017.9.1.4 
adding new services that consume more bandwidth. New business models, such as HDTV (which needs $20 \mathrm{Mbit} / \mathrm{s}$ per channel), online game, interactive E-learning, broadcast TV and radio as well as video on demand will dramatically increase bandwidth demand. Recently [1,2], the PON technology development is highly diverse. For the examples are the GPON and XGPON which have bite rate up to $2.5 \mathrm{Gbps}$ and $10 \mathrm{Gbps}$ respectively. To keep safe existing investment of Telco operators, an investigation should be done to make sure that those technologies can be co-operate and co-existence in some location.

\section{Passive Optical Networks}

Optical Network [3] is telecommunication infrastructure with highest bandwidth capacity and on the other hand lowest attenuation characteristics. It makes it suitable to transmit broadband communication services such as gigabit and terabit transmission.

Basically optical network consists of transmitter, transmission channel and receiver. Transmitter contains light source and transmitter circuit. The transmission channel consists of optical cable, regenerator, splitter, coupler, multiplexer, connector. In the receiver there are photo detector, amplifier and receiver circuit.

In the last mile, optical access network reach to any home uses PON that extend from an operator central office into individual homes, apartment houses and business offices. It uses passive optical splitters to split fiber constructing tree network topologies. The general structure of a PON network is shown in Figure 1.

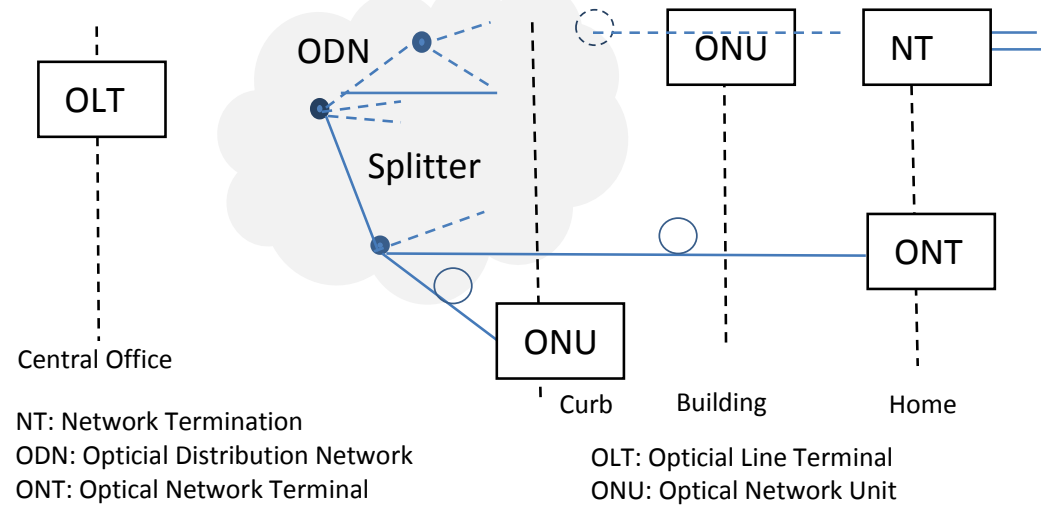

Figure 1. Structure of Passive Optical Network

The basic elements of PON are an optical line terminal (OLT) at the central office side and a number of optical network units (ONUs) or Optical Network Terminals (ONTs) near end customers premise. The OLT acts as an interface between all users in the given PON and the other network. Users access the services through a network terminal (NT) connected to the optical distribution network (ODN) via the ONU/ONTs. ODN builds a tree network topology by using a point-to-multipoint configuration with splitters. PON splitters usually are placed close to the user sites and typically has splitting ratios of 1:16, 1:32 and 1:64 depends on number of user will be covered.

ITU-T G652 Recommendation supports PON splitter with split ratio 16, 32 or 64 way to split. In order to increase income per central office, operators try to increase subscriber density per OLT port, using high split systems and maximizing take ratios, so the larger the split ratio $(1: 64)$ is the more attractive for operators.

\section{A. PON Evolution}

In the early stage, APON (ATM PON) [4] based on the Asynchronous transfer protocol technology uses the older ITU-T G.983 standard. It delivers STM-1 (155 Mbps) bandwidth on a single fiber, due to the high performance of ATM technology. 
In 2004, EPON [5] based on Ethernet protocol technology uses IEEE 802.3 standard which delivers symmetric bandwidths with 1 Gbps upstream and downstream rates. It is well known that the Ethernet protocol is applicable for data-centric networks, including voice, data and video networks services.

Final version of ITU-T G.983x, being referred to broadband PON, is called BPON [6]. BPON is enhancement of an APON with broadband services capabilities. It provides downstream bandwidth for STM-4 data rates (622 Mbps) and STM-1 data rates (155 Mbps) for upstream..

GPON is based on the ITU-T G.984x [7,8] Gigabit Passive Optical Networks standard. It has more bandwidth capability compared to BPON, in term of the total bandwidth, bandwidth efficiency and variable-length packets. It is being deployed by the operators to meet their Ethernet and Voice and TDM services' needs. The standards provides data rate on STM-16 (2.5 Gbps) for downstream bandwidth, and STM-8 (1.25 Gbps) for upstream bandwidth. It has been defined as full services by the huge deployment of PON networks worldwide.

Finally, G.987x defined XG-PON [9-17]. It offers STM-64 (10 Gbps) for downstream and STM-16 (2.5 Gbps) for upstream. Asymmetric XG-PON is specified as XG-PON1, while Symmetric XG-PON is also proposed as XG-PON2. It's framing is similar to GPON and consist of service support capabilities as well as enhanced bandwidth and performance of access nodes. The guidance of PON development is a key factor for Telcos, especially to provide the next broadband services.

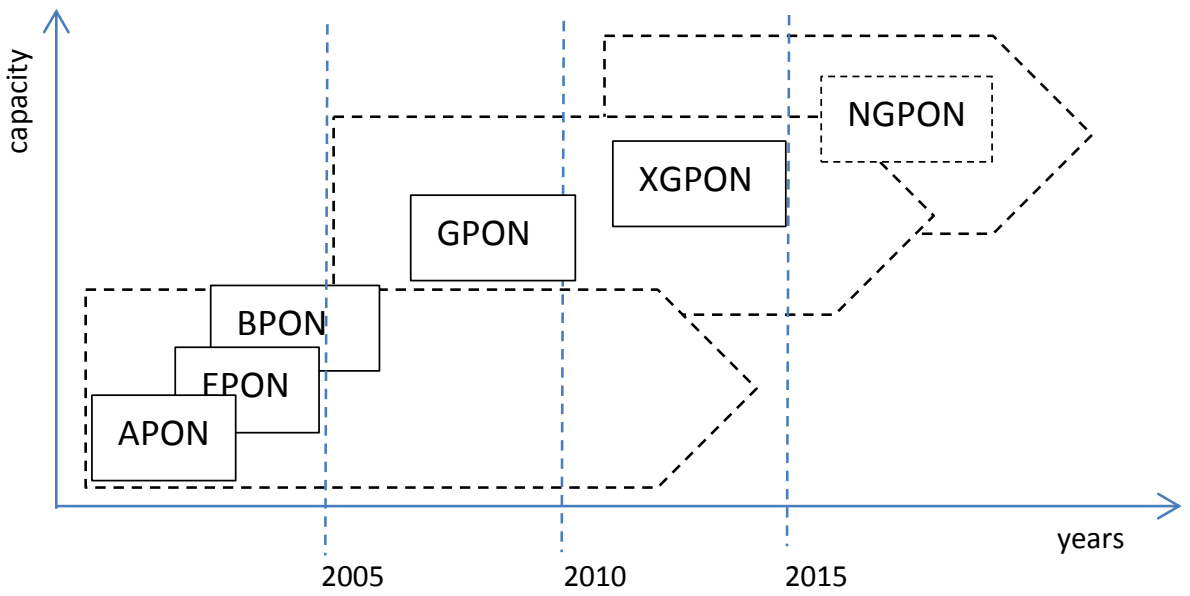

Figure 2. Evolution of PONs Technologies

\section{B. Hybrid GPON and XGPON}

In general, GPON, XGPON and hybrid technologies [18] have similar configuration. They consists of an optical line terminal (OLT) at the originating service provider, transmission channel which consist of optical cable and splitter, and a number of optical transmitter, network units (ONUs), near end user premises. The performance of GPON and XGPON with splitting ratio 1:64 in Indonesia has been reported in [8] and [12] respectively. This paper analyzes the performance of the hybrid GPON and XGPON that will be implemented in Indonesia. General block diagram of the topology of hybrid GPON and XGPON using 1:64 splitter is shown on figure 3 .

\section{B.1. Optical Line Terminal}

The OLT consists of downstream transmitter and upstream receiver. Combining these two technologies requires separate transmitter and receiver components between GPON and XGPON. GPON transmitter uses DFB laser with $8 \mathrm{dBm}$ power and a wavelength of $1490 \mathrm{~nm}$. The modulation process uses a Mach-Zehnder external modulator with NRZ coding and 
extinction ratio of $25 \mathrm{~dB}$. The XGPON transmitter uses DFB laser with $12.5 \mathrm{dBm}$, the wavelength of $1577 \mathrm{~nm}$, NRZ coding modulator and extinction ratio of $25 \mathrm{~dB}$. The second signal is amplified by a $20 \mathrm{~dB}$ optical amplifier and then merged using WDM1r or WDM combiner to multiplex the signals with different wavelengths.

GPON upstream receiver uses a PIN photodetector with a sensitivity of $-28 \mathrm{dBm}$, and a low pass filter with a cutoff frequency in the period of $1310 \mathrm{~nm}$. The XGPON upstream receiver uses a PIN photodetector with a sensitivity of $-29.5 \mathrm{dBm}$ and a low pass filter in the period of $1270 \mathrm{~nm}$. The signal from the fiber optic cable is separated first by the WDM demultiplexer then received by each receiving device. Detail circuit diagram of OLT Hybrid is shown in Figure 4.

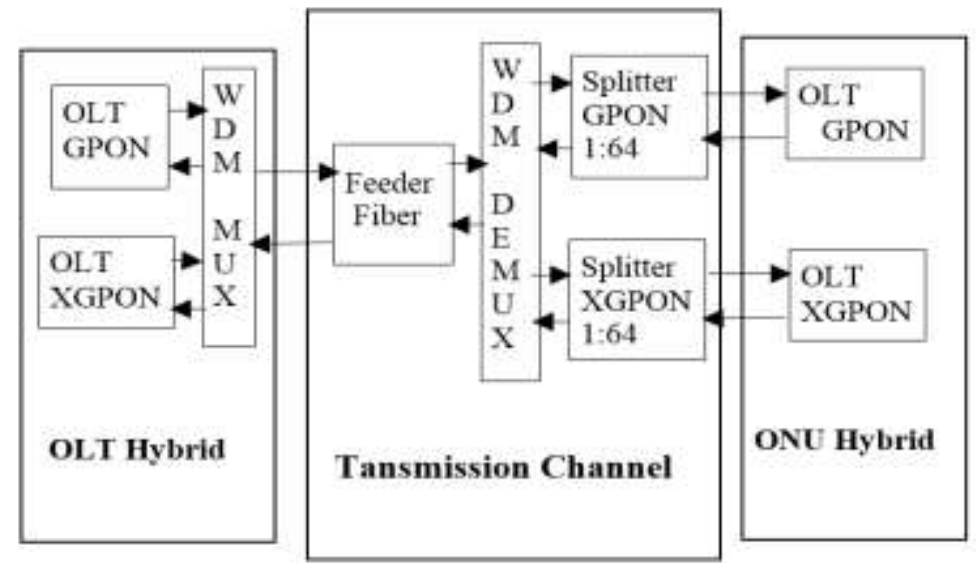

Figure 3. Block diagram of hybrid GPON-XGPON Topology

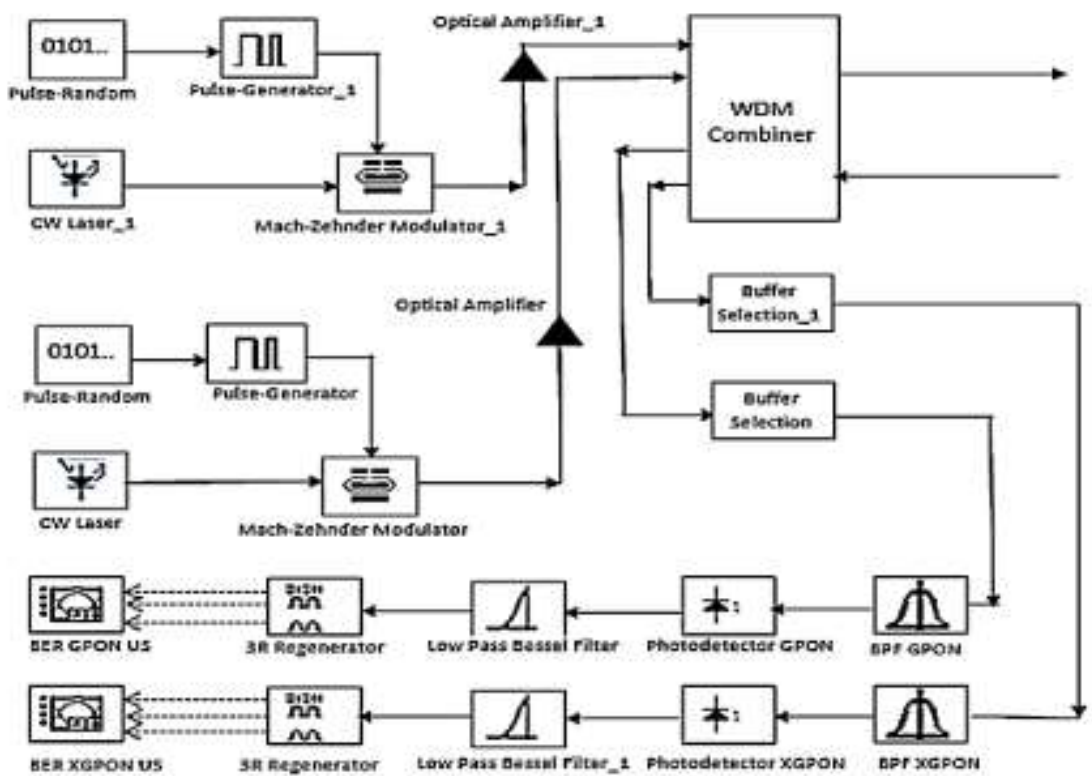

Figure 4. The detail circuit of OLT Hybrid

\section{B.2. Transmission Channel.}

The transmission channel on GPON-XGPON hybrid system uses the optical fibers in accordance to ITU-T G.652 standard. Constraints are applied using a worst case condition according to the highest wavelengths, ie, XGPON wavelengths. The worst cable attenuation according to the ITU-T G.652 standard is $0.47 \mathrm{~dB} / \mathrm{km}$ at $1260 \mathrm{~nm}$ wavelength. And the 
maximum length of the cable is $20 \mathrm{~km}$. The 1:64 splitter places on the remote node. Detail diagram of hybrid transmission channel is shown on Figure 5.

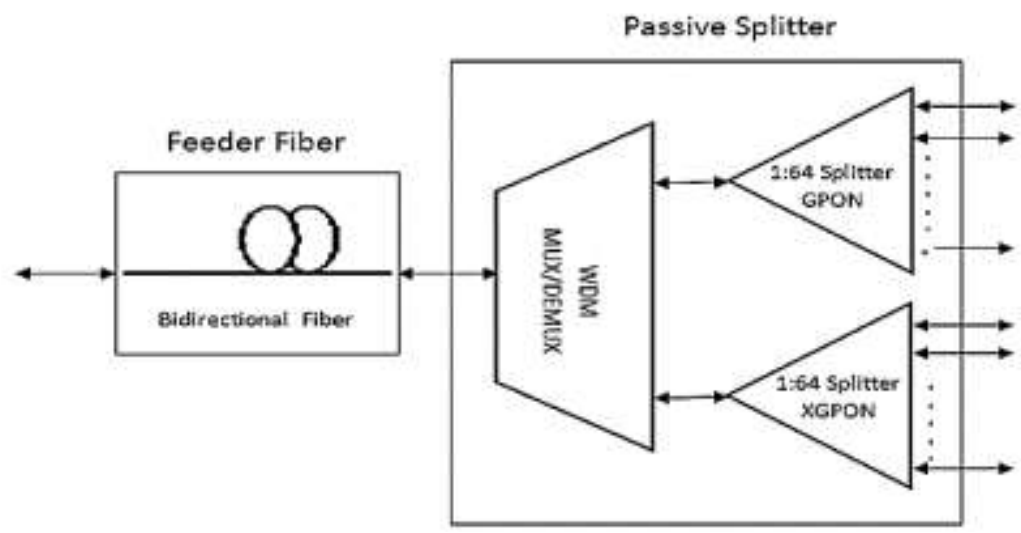

Figure 5. The Hybrid transmission channel
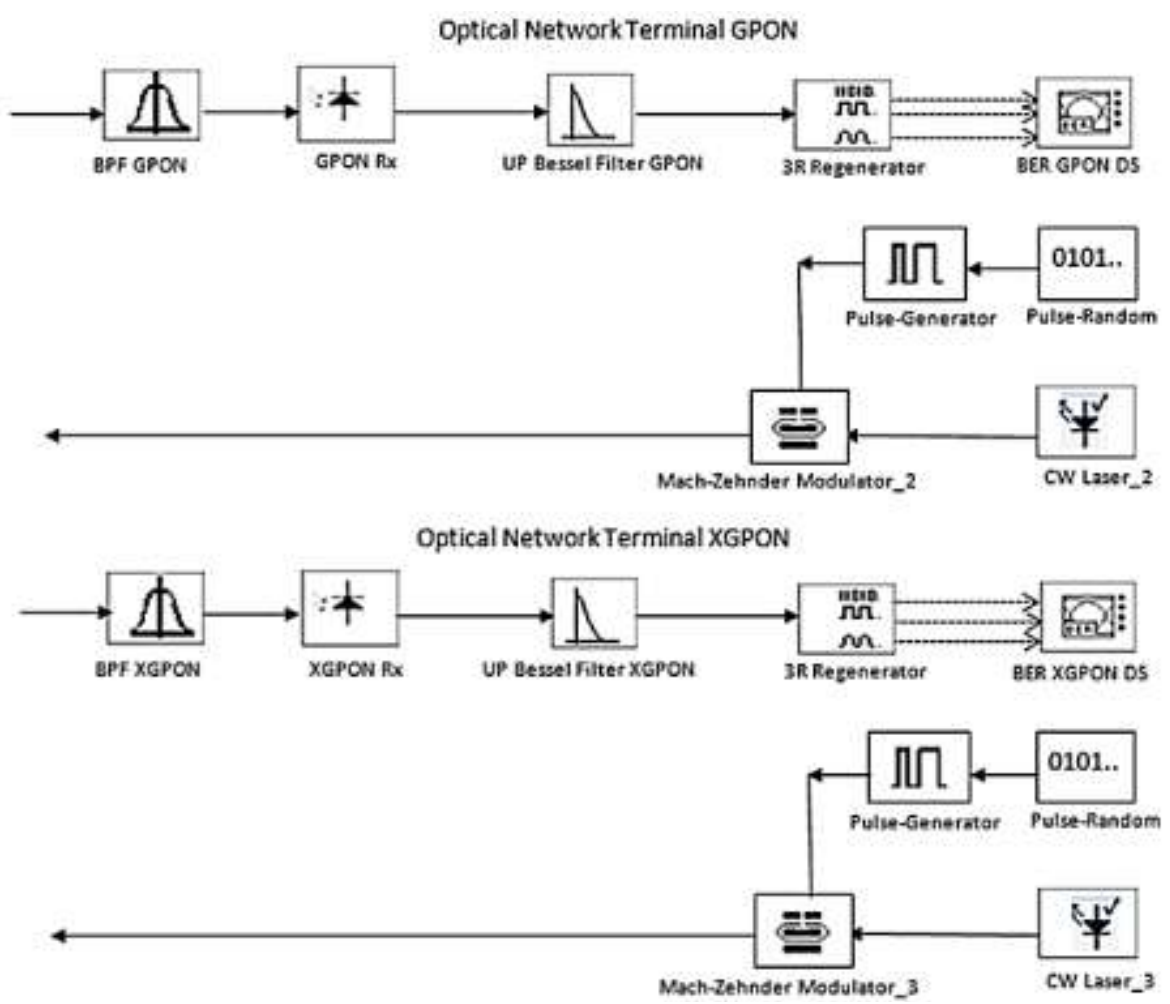

Figure 6. Hybrid Optical Network Unit

\section{B.3 Optical Network Unit (ONU).}

ONU at the receiver side consists of a upstream transmitter and downstream receiver. Signals from the fiber optic cable are separated by WDM demultiplexer, and detected by the receiving devices of each technology. The GPON downstream receiver uses a PIN photodetector with $-21 \mathrm{dBm}$ sensitivity. The detected signals are then fed into a low pass filter to obtain the information signal. While the GPON upstream transmitter uses DFB laser with 3 $\mathrm{dBm}$ of power, NRZ coding and modulator with has $25 \mathrm{~dB}$ extinction ratio. 
The XGPON downstream receiver uses a PIN photodetector with $-21.5 \mathrm{dBm}$ sensitivity and a low pass filter with $1577 \mathrm{~nm}$ cutoff frequency to obtain the information signal. The XGPON Upstream transmitter uses a DFB laser with $7 \mathrm{dBm}$ power, NRZ coding and modulator with 25 $\mathrm{dB}$ extinction ratio. The signal is also amplified by the $20 \mathrm{~dB}$ optical amplifier Gain. Detail diagram of ONU Hybrid is shown in Figure 6.

\section{PON Performance Parameters}

Two budget parameters give necessary conditions for satisfactory performance operation of optical network, those are link power budget and rise time budget. To evaluating the performance of digital transmission, the eye pattern method has been used extensively.

\section{C.1 Link Power Budget}

In order to operate properly, a fiber optic network link must have enough power margin in the system to meet the given bit error rate. That is, the total loss in the installed cable plant must be less than the tolerable loss of the transmitters and receivers in the transmission equipment being used. Figure 7 graphically illustrates the link loss parameters includes all items in the cable plant, such as fiber length, number of connectors and splices, and any other passive components such as optical splitters. Equation 2.1 shows the relationship between those parameters.

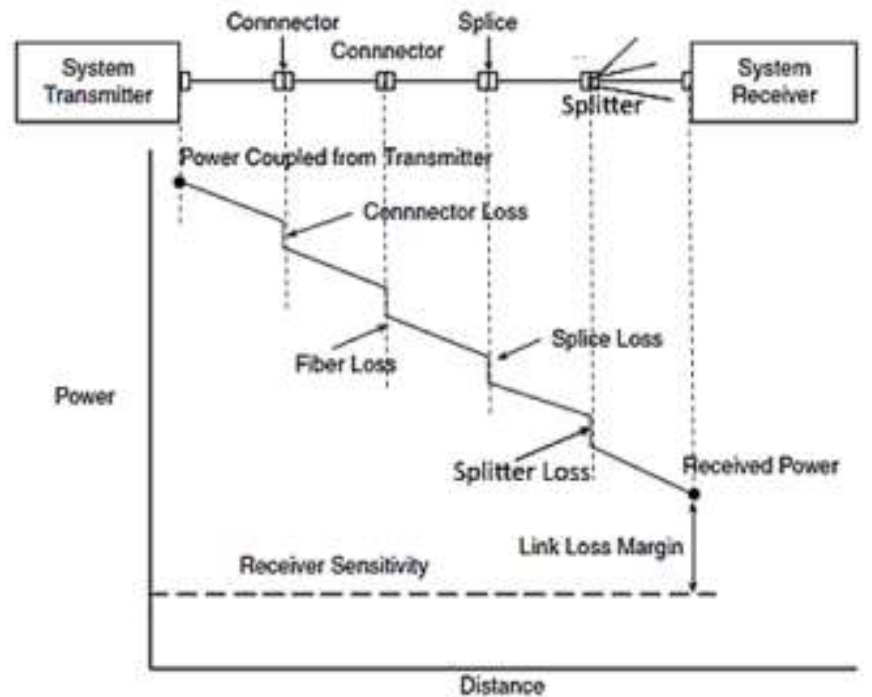

Figure 7. Link Loss Parameters

$$
\mathrm{P}_{\mathrm{T}}=\mathrm{P}_{\mathrm{S}}-\mathrm{P}_{\mathrm{R}}=\mathrm{ml}_{\mathrm{c}}+\mathrm{nl}_{\mathrm{sp}}+\alpha_{\mathrm{f}} \mathrm{L}+\mathrm{ol}_{\text {split }}+\text { Link Loss Margin }
$$

$\mathrm{P}_{\mathrm{T}}$ : Total loss, $\mathrm{P}_{\mathrm{S}}$ : Source power, $\mathrm{P}_{\mathrm{R}}$ : Receiver sensitivity, $\mathrm{ml}_{\mathrm{c}}$ : Connector loss, $\mathrm{nl}_{\mathrm{sp}}$ : Splice loss, $\alpha_{\mathrm{f}} \mathrm{L}$ : Cable loss, $\mathrm{l}_{\text {split }}$ : Splitter loss. According to the ITU-T recommendation, the link loss margin is $5 \mathrm{~dB}$.

\section{C.2 Rise Time Budget}

A rise time budget analysis is a convenient method for determining the dispersion limitation of an optical link. That is, a link on an optical network should fast enough to meet the given bit rate. The total rise time depends on transmitter rise time $\left(t_{t x}\right)$, group velocity dispersion $\left(\mathrm{t}_{\mathrm{gvd}}\right)$, modal dispersion rise time $\left(\mathrm{t}_{\mathrm{mod}}\right)$ and receiver rise time $\left(\mathrm{t}_{\mathrm{rx}}\right)$. Equation 2.2 
describes the rise time total system. In a digital link, the rise time should not exceed $70 \%$ for a NRZ bit period, and $35 \%$ of a RZ bit period [3].

$$
\mathrm{T}_{\mathrm{sys}}=\left[\mathrm{t}_{\mathrm{tx}}{ }^{2}+\mathrm{t}_{\mathrm{gvd}}{ }^{2}+\mathrm{t}_{\mathrm{mod}}{ }^{2}+\mathrm{t}_{\mathrm{rx}}{ }^{2}\right]^{1 / 2}
$$

\section{C.3 Eye Pattern}

The eye pattern technique is a simple but powerful measurement method that has been broadly used for evaluating the performance of optical fiber data links. The eye pattern measurement are made in time domain and allow the effects of waveform distortion to be shown immediately on an oscilloscope. A great deal of system performance information can be deduced from the eye pattern display. It can indicate the best point for sampling, divulge the signal-to-noise ratio at the sampling point, and indicate the amount of jitter and distortion. Additionally, it can show the time variation at zero crossing, which is a measure of jitter. Figure 8 shown simplified drawing of eyes diagram.

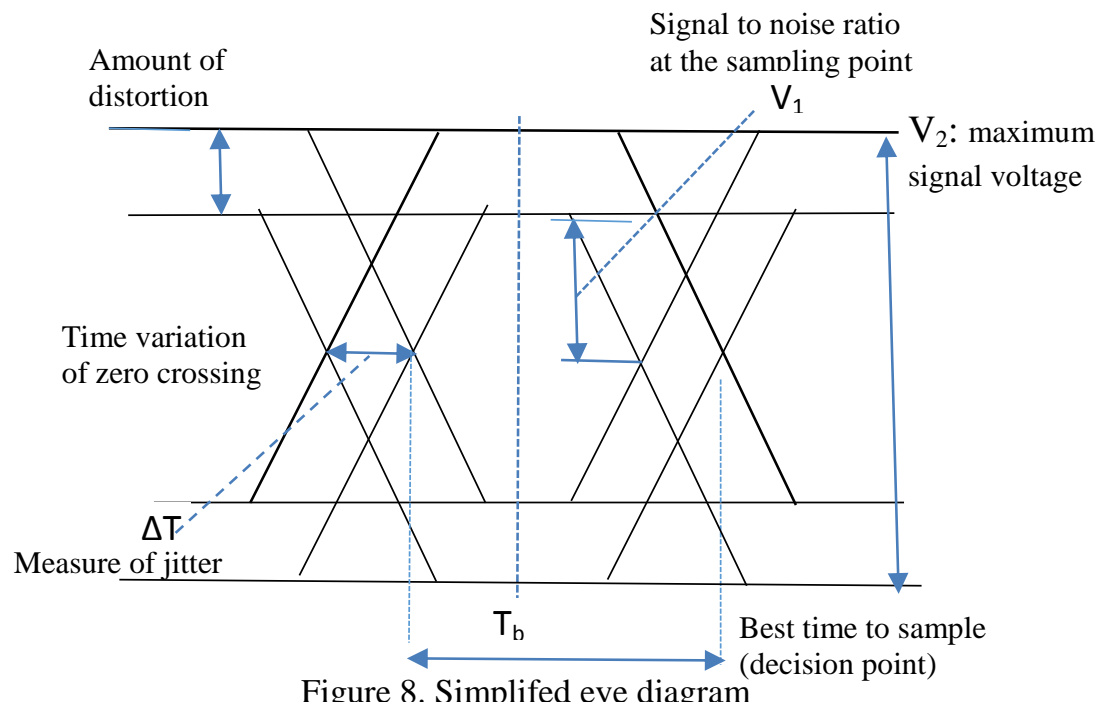

Figure 8. Simplifed eye diagram

$$
\begin{aligned}
& \text { Timing Jitter }(\text { percent })=\left(\Delta \mathrm{T} / \mathrm{T}_{\mathrm{b}}\right) \times 100 \text { percent } \\
& \text { Noise Margin }(\text { percent })=\left(\mathrm{V}_{1} / \mathrm{V}_{2}\right) \times 100 \text { percent }
\end{aligned}
$$

\section{Simulation and Analysis}

The performance analysis calculates the link budget and rise-time budget of the end-to-end system to see whether these parameters meet the requirements of the basic quality of the network connection. Link budget calculation determines whether sufficient power meet the receiver sensitivity, while rise time budget measures the feasibility of the signal shape and dispersion effect. We simulate the design using Opti-system software to find its feasibility. The parameters used in the simulation are the power received in each component, the ratio of the signal shape in the receiver and the sender, BER and eye diagram of each receiver.

\section{A. Link Budget}

GPON-XGPON hybrid configuration has two separate sections for each of the technologies at the transmitter and receiver. link budget observations is done by observing each transmitter and receiver output of each technology component. 
From figure 9 (a) and (b), we can see the results of power measurement of GPON downstream transmitter power is at $4.4 \mathrm{dBm}$ and receiver power is at $-19.3 \mathrm{dBm}$. It means that the power loss during transmission is $22.9 \mathrm{~dB}$. Then, the received power is above the receiver device sensitivity of which is $-21 \mathrm{dBm}$. So, there is only $1.7 \mathrm{~dB}$ of margin. We can conclude that that, link budget-wise, GPON downstream on the hybrid configuration is feasible, but not good enough because of small margin.

From figure 10 (a) and (b), we can see the results of power measurement of XGPON downstream transmitter power is at $9.5 \mathrm{dBm}$ and receiver power is at $-18.2 \mathrm{dBm}$. It means that the power loss during transmission is $27.7 \mathrm{~dB}$. The received power is above the receiver device sensitivity, which is $-21.5 \mathrm{dBm}$. In this case we have $3.3 \mathrm{~dB}$ of margin. We can conclude that that, link budget-wise, XGPON downstream on the hybrid configuration is feasible, but still not good enough because of small margin.

At the upstream side, the link budget analysis is done by observing the transmitter power at ONU and the received power at OLT. Figure 11-12 show the observed power.

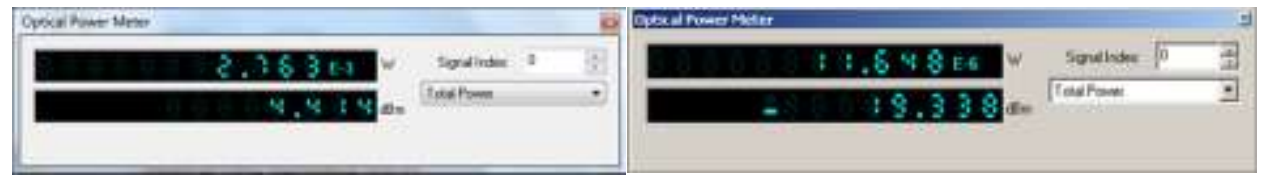

Figure 9. Power of GPON downstream transmitter (a) and receiver (b) of the Hybrid

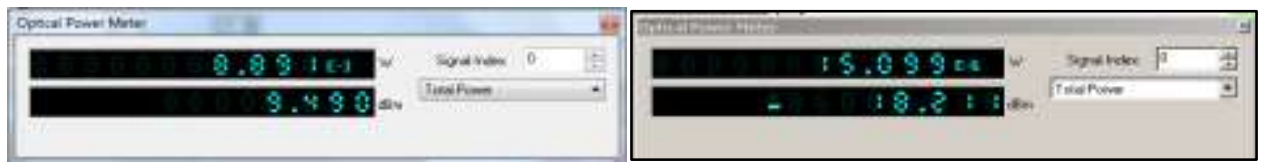

Figure 10. Power of XGPON downstream transmitter (a) and receiver (b) of the Hybrid

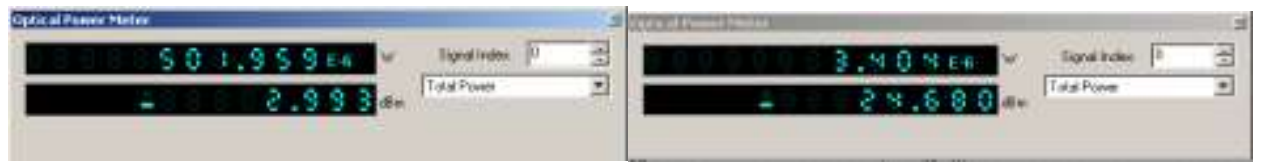

Figure 11. Power of GPON upstream transmitter (a) and receiver (b) of the Hybrid

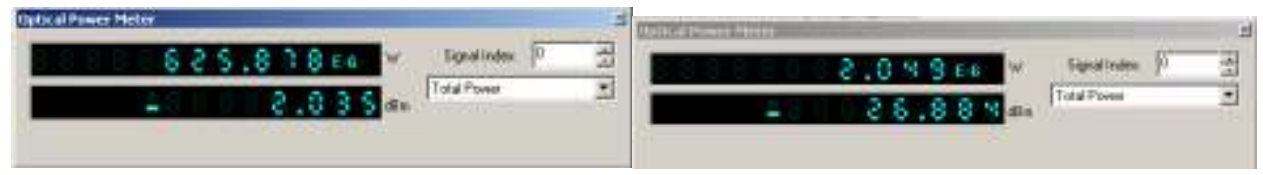

Figure 12. Power of XGPON upstream transmitter (a) and receiver (b) of the Hybrid

From figure 11 (a) and (b), power measurement of the GPON upstream, we can see that transmitter power is $-2.99 \mathrm{dBm}$ and received power is $-24.68 \mathrm{dBm}$. It means that the power loss is $21.69 \mathrm{~dB}$ during transmission. The received power is above the receiver device sensitivity, which is $-28 \mathrm{dBm}$. There is $3.3 \mathrm{~dB}$ for margin. Then, GPON upstream on the hybrid configuration is also feasible, but also still not good enough because of small margin.

Figure 12 (a) and (b) show power measurement of the XGPON upstream, transmitter power is $-2 \mathrm{dBm}$ and received power is $-26.9 \mathrm{dBm}$. It means the power loss is $24.9 \mathrm{~dB}$ during transmission. The received power is above the receiver device sensitivity, which is $-29.5 \mathrm{dBm}$. There is a $2.6 \mathrm{~dB}$ for margin. So, XGPON upstream on the hybrid configuration is feasible, but not good enough because of small margin. Noted, the minimal margin is $5 \mathrm{~dB}$.

\section{B. Rise-time Budget}

Rise time budget calculation in hybrid configuration is performed on each output for each technology. It is done by observing the signal shape at the transmitter and receiver. Rise-time 
budget is viable if the rise-time changes do not exceed $70 \%$ of the original time. Rise-time values can be observed from the time it takes to rise from 10 up to $90 \%$ of the maximum value. Connection is feasible if the rise-time value variation does not exceed $70 \%$ of the signal period. In the GPON connection, with $2.5 \mathrm{Gbps}$ of bit rate, the signal bit period is calculated as follow:

$$
T_{b}=\frac{1}{2,5 \times 10^{9}}=0,4 n s
$$

So the maximum rise-time variation allowed is $70 \% \mathrm{~Tb}$, which is $0.7 \times 0.4=0.28 \mathrm{~ns}$.

In the XGPON connection, with $10 \mathrm{Gbps}$ of bit rate, the signal bit period is calculated as follow:

$$
T_{b}=\frac{1}{10 \times 10^{9}}=0,1 \mathrm{~ns}
$$

So the maximum rise-time variation allowed is $70 \% \mathrm{~Tb}$, which is $0.7 \times 0.1=0.07 \mathrm{~ns}$. Observed signals form on the GPON downstream are shown in Figure 13 (a) and (b), while on the GPON upstream side are shown in Figure 14 (a) and (b).$$
\text { GPON TXDS }
$$

GPON DS Final Signal
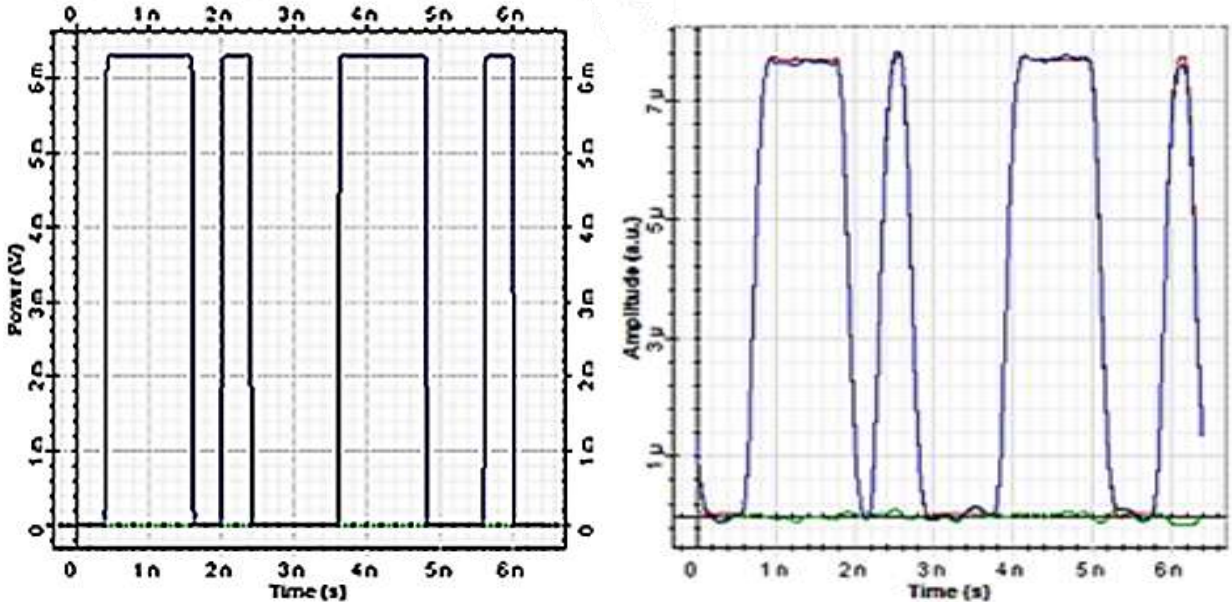

Figure 13. The GPON downstream (a) transmit and (b) receive signal on the hybrid topology

GPON TX US

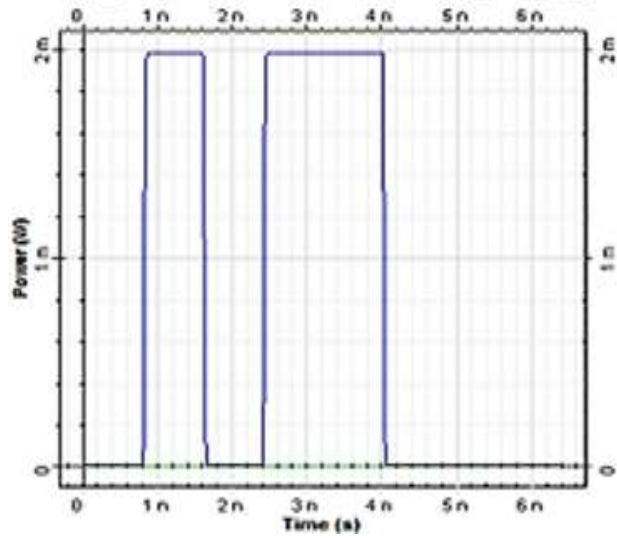

GPON US Final Signal

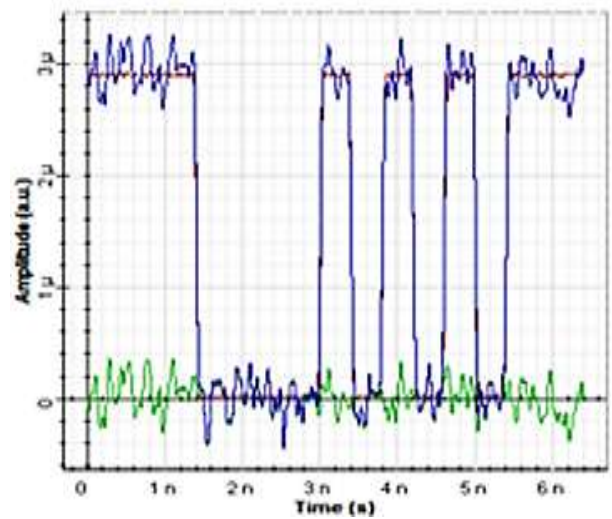

Figure 14. The GPON upstream (a) transmit and (b) receive signal on the hybrid topology 
Figure 13 (a) and (b), show that the received signals shape resembles the information signal from the transmitter. The reading of the received signal will produce a bit of information similar to that emitted by sender. Based on GPON transmitter observation, we have $0,01395 \mathrm{~ns}$ of rise time, and the receiver rise time is $0,19087 \mathrm{~ns}$. Both of the values are smaller than the maximum change in GPON rise time, which is $0.28 \mathrm{~ns}$. So, in the downstream connection, the GPON declared eligible.

In the similar way for the GPON upstream side, based on Figure 14 (a) and (b), the received signal will produce a bit of information similar to that emitted by sender. Observed rise time on the transmitter at the ONU is $0.028 \mathrm{~ns}$ and at the receiver in the OLT is $0.050 \mathrm{~ns}$. Both of the values are smaller than the maximum change in GPON rise time, which is $0.28 \mathrm{~ns}$. So, in the upstream connection, the GPON also is feasible.

XGPON TXDS



XGPON DS Final Signal

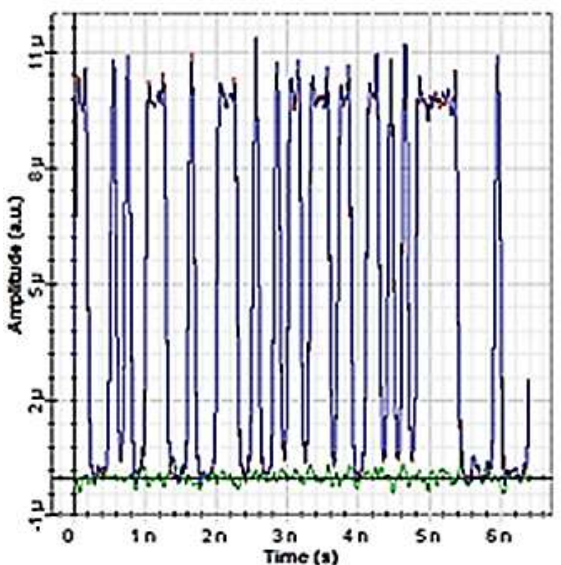

Figure 15. The XGPON downstream (a) transmit and (b) receive signal on the hybrid topology

XGPON TXUS

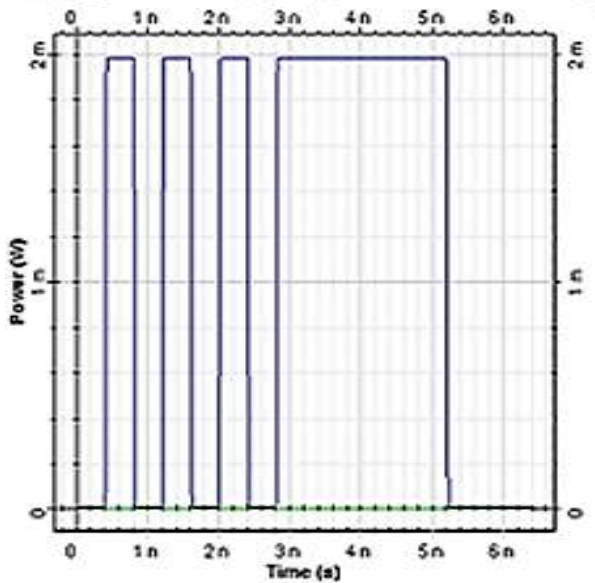

XGPON US Final Signal



Figure 16. The XGPON upstream (a) transmit and (b) receive signal on the hybrid topology

Observed signals form on the XGPON downstream side are shown in Figure 15(a) and (b), while on the XGPON upstream side are shown in Figure 14 (a) and (b). Based on Figure 15 (a) and (b), the shape of the received signals resembles the information signal from the transmitter. The reading of the received signal will produce a bit of information similar to that emitted by 
sender. Observed XGPON transmitter rise time is $5,269 \times 10^{-3} \mathrm{~ns}$, and the receiver rise time is $0,05957 \mathrm{~ns}$. Both of these values are smaller than the maximum change in XGPON rise time, which is 0.07 ns. In similar way, for the upstream side, based on Figure 16 (a) and (b), the shape of the received signals resembles the information signal from the transmitter. Observed XGPON transmitter rise time is $0,0139 \mathrm{~ns}$, and the receiver rise time is $0,0506 \mathrm{~ns}$. Both of these values are smaller than the maximum change in XGPON rise time, that is $0.07 \mathrm{~ns}$.

\section{BER Analysis}
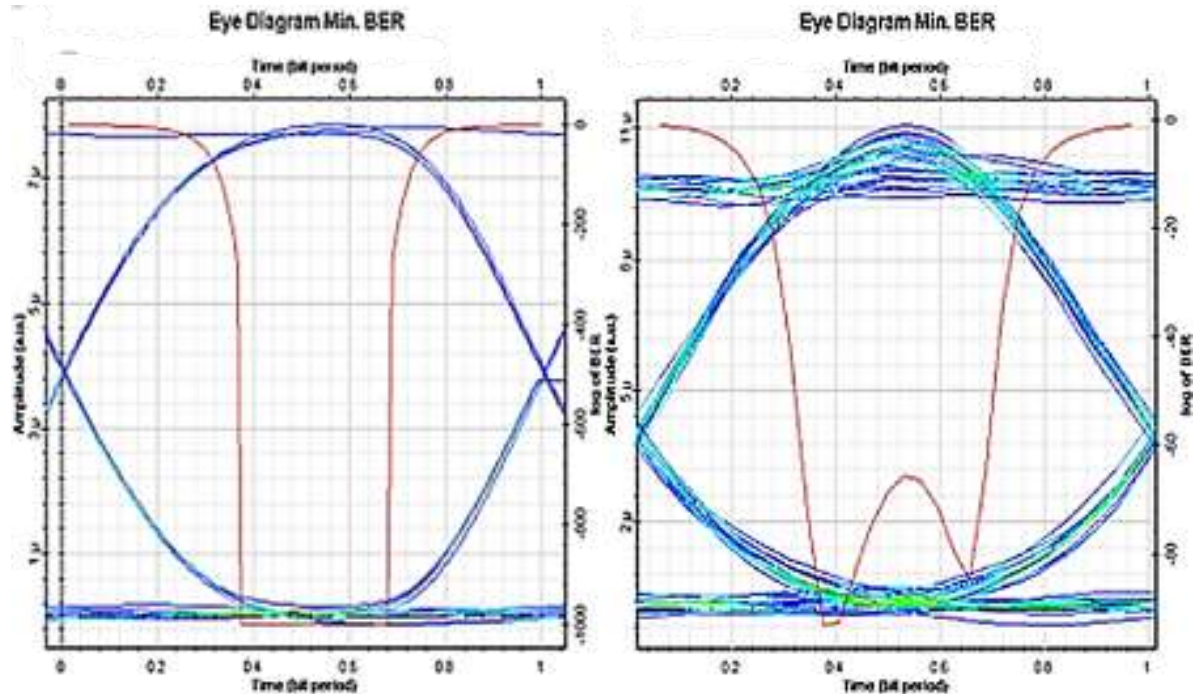

Figure 17. BER of downstream (a) The GPON and (b) The XGPON on the hybrid topology
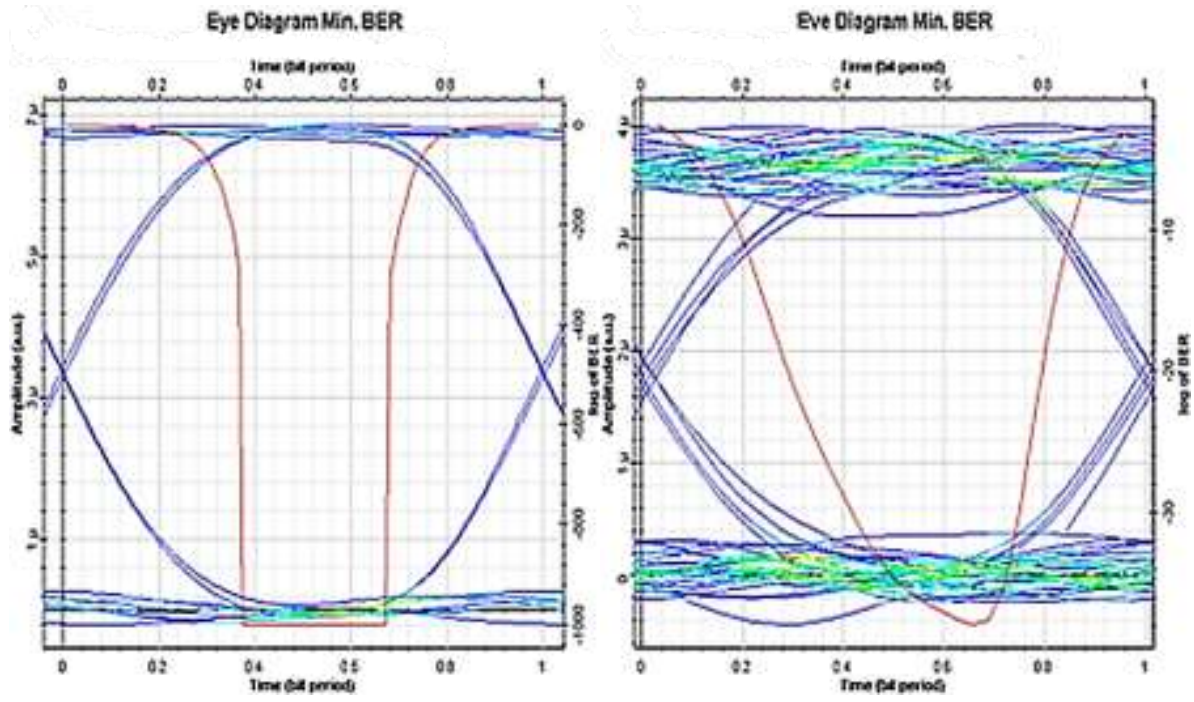

Figure 18. BER of upstream (a) The GPON and (b) The XGPON on the hybrid topology

The BER Analyzer generates the eye diagram on the output downstream and the output upstream. Using the eye diagram, the distortion, the jitter and the BER can be determined. BER analysis observes the output value of BER and eye diagrams on each output component of the receiver. In GPON-XGPON hybrid configuration, analysis is conducted for each 
receiver output of each technology. At the downstream side, the output of BER Analyzer observed as the "BER GPON DS" in the Figure 17(a) and "BER XGPON DS" in the Figure 17(b). On the upstream side, the output BER Analyzer observed as the "BER GPON UP" in the Figure 18(a) and "BER XGPON UP" in the Figure 18(b).

Eye diagram, obtained from BER Analyzer, is very clear for both of downstream and upstream, It means the communication performance of the GPON-XGPON hybrid configuration is very good. In term of distortion and jitter, the eye diagram of the signal in downstream side is better than the upstream side. These distortion and jitter are very small so that communication can be declared eligible for GPON-XGPON hybrid configuration.

Table 1. The performance of Hybrid GPON and XGPON

\begin{tabular}{|c|c|c|c|c|c|c|c|c|c|c|}
\hline \multirow{2}{*}{\multicolumn{2}{|c|}{ Configuration }} & \multicolumn{2}{|c|}{$\begin{array}{c}\text { DS power } \\
\text { Received }(\mathrm{dBm})\end{array}$} & \multicolumn{2}{|c|}{$\begin{array}{c}\text { US power } \\
\text { Received }(\mathrm{dBm})\end{array}$} & \multicolumn{3}{|c|}{$\begin{array}{c}\text { Rise time } \\
\text { (ns) }\end{array}$} & \multicolumn{2}{|c|}{ Eyes diagram } \\
\hline & & Thrsld & Power & Thrsld & Power & Thrsld & $\mathrm{Tx}$ & $\mathrm{Rx}$ & DS & US \\
\hline \multirow[t]{2}{*}{ Hybrid } & GPON & -21 & -19.3 & -28 & -24.7 & 0.28 & 0.01 & 0.19 & $\begin{array}{l}\text { Very } \\
\text { Good }\end{array}$ & $\begin{array}{l}\text { Very } \\
\text { Good }\end{array}$ \\
\hline & XGPON & -21.5 & -18.2 & -29.5 & -26.8 & 0.07 & 0.03 & 0.05 & Good & Good \\
\hline
\end{tabular}

\section{Conclusion}

The optical communication system using GPON-XGPON hybrid configuration technology with splitting ratio of 1:64 proposed in this work can be implemented in FTTH to provide broadband services in Indonesia. The simulation results shows that based on the link budget analysis and measurement, the received power at GPON downstream and upstream receivers are $-19.3 \mathrm{dBm}$ and $-24.7 \mathrm{dBm}$ respectively. The received power at XGPON downstream and upstream receivers are $-18.2 \mathrm{dBm}$ and $-26.8 \mathrm{dBm}$ respectively. Those values are above the sensitivity threshold of receiver components. Rise-time and BER analysis show some very small distortions with easy to read eye diagrams at the outputs. Its can be concluded that the migration from GPON to XGPON in Indonesia can be done smoothly via hybrid mode to keep safe existing investment of Telco operator.

\section{References}

[1]. K.Helsen et.al, "FTTH Business Guide", Version 1.0 9, FTTH Council Europe 2010.

[2]. S.B. Weinstein et al., "The Comsoc Guide to Passive Optical Networks", NJ: John Wiley \& Sons Inc., 2012.

[3]. G. Agrawal, "Fiber-Optic Communication Systems". New York: Wiley, 2002.

[4]. M. Kudo et al.,"ATM-PON for Optical Communication Transmission/Reception Optical Module", OKI Tech. Review vol. 68, pp 64-67, Maret 2001.

[5]. G. Kramer and G.Pesavento, "Ethernet Passive Optical Network (EPON): building a next generation optical access network", IEEE,Vol 40,Issue 2, pp. 66-73, 2002.

[6]. D. Hood and L.Lu, "Current and Future ITU-T PON Systems and Standards", 17th OptoElectronics and Communications Conference, 2012.

[7]. "Gigabit-capable Passive Optical Networks (GPON): Physical Media Dependent (PMD) layer specification”, ITU-T G.984.2, 2003.

[8]. N.R. Syambas and F. Rahadian, "Performance Analysis of Gigabit Passive Optical Network with Splitting Ratio of 1:64", Int.Conf. on Wireless and Telematics, ICWT, 2015.

[9]. K. Tanaka, et al., "IEEE 802.3av 10G-EPON Standardization and Its Research and Development Status”, Lightwave Technology, Journal of, vol. 28, pp. 651-661, 2010.

[10]. "10-Gigabit-capable Passive Optical Networks (XG-PON): Physical Media Dependent (PMD) Layer Spesification”, ITU-T G.987.2, 2010. 
[11]. S. Jain et al., "World's First XG-PON Field Trial," J. Lightw. Technol., vol. 29, no.4, 2011.

[12]. N.R. Syambas and. Rahadian, "Performance Analysis of 10-Gigabit Passive Optical Network with Splitting Ratio of 1:64", Int. Conf. on Wireless and Telematics, TSSA, 2015.

[13]. Y. Lee et al., "Fast Management of ONUs Based on Broadcast Control Channel for a 10 Gigabit-Capable Passive Optical Network (XG-PON) System," J. Commun. And Networks, vol. 15, no. 5, pp. 538-542, Oktober 2013.

[14]. A.Kaur et al, "Performance Analysis of 10G/2.5G Asymmetric XGPON Transmission using RZ and NRZ Data Formats", International Journal of Computer Applications (0975-8887) Volume 70, No.15, May 2013.

[15]. Y. Lee et al., "Fast Management of ONUs Based on Broadcast Control Channel for a 10 Gigabit-Capable Passive Optical Network (XG-PON) System”, J. Commun. And Networks, vol. 15, no. 5, pp. 538-542, October 2013.

[16]. J. Kim et al., Design and Performance Analysis of Passively Extended XG-PON with CWDM Upstream, J. Lightw. Technol., vol. 30, no. 11, pp. 1677-1684, June 2012.

[17]. F. J. Effenberger, "The XG-PON System: Cost Effective 10 Gb/s Access", J. Lightw. Technol., vol. 29, no. 4, pp. 403-409, Feb. 2011.

[18]. B. Zhu et al., "Coexistence of 10G-PON and GPON Reach Extension to 50-km with Entirely Passive Fiber Plant", Optical Society of America, 2011.

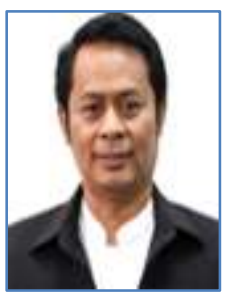

Nana Rachmana Syambas. He was graduated from his bachelor degree at Electrical Engineering Department, ITB in 1983. He got his Master by Research degree from Royal Melbourne Institute of Technology, Australia in 1990 and doctoral degree from School of Electrical Engineering and Informatics, ITB in 2011. He has been a lecturer at School of Electrical Engineering and Informatics, ITB since 1984. His research interest includes: Telecommunication Networks, Telematic Services, Software defined network (SDN), Named Data Network (NDN), Protocol engineering and Tele-traffic engineering. He has authored or coauthored over 50 published articles.

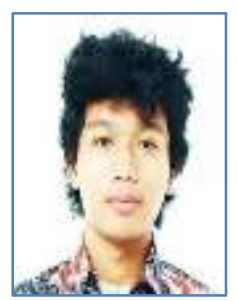

Rahadian Farizi. He was graduated from his bachelor of engineering degree at School Electrical Engineering and Informatics, ITB in 2015. He has been a honorary staff of outside plant division at PT.QDC since 2015. His research interest includes: optical network, outside plant and fiber optic to the home. 